\title{
Geometric phases in open systems: an exact model to study how they are corrected by decoherence
}

\author{
Fernando C. Lombardo ${ }^{*}$ and Paula I. Villar ${ }^{\dagger}$ \\ Departamento de Física Juan José Giambiagi, FCEyN UBA, Facultad de Ciencias Exactas y Naturales, \\ Ciudad Universitaria, Pabellón I, 1428 Buenos Aires, Argentina
}

\begin{abstract}
We calculate the geometric phase for an open system (spin-boson model) which interacts with an environment (ohmic or nonohmic) at arbitrary temperature. However there have been many assumptions about the time scale at which the geometric phase can be measured, there has been no reported observation of geometric phases for mixed states under nonunitary evolution yet. We study not only how they are corrected by the presence of the different type of environments but estimate the corresponding times at which decoherence becomes effective as well. These estimations should be taken into account when planning experimental setups to study the geometric phase in the nonunitary regime, particularly important for the application of fault-tolerant quantum computation.

PACS numbers: 03.65.Vf;03.65Yz;03.67.Pp;03.75Dg
\end{abstract}

\section{INTRODUCTION}

All real world quantum systems interact with theirs surrounding environment to a greater or lesser extent. While closed quantum systems are bound to have an unitary evolution in which the system's purity is preserved and the superposition principle can be applied, open quantum systems show a different scenario. As they are in interaction with an environment (defined as any degrees of freedom coupled to the system which can entangle its states), a degradation of pure states into mixtures takes place. These mixtures states will often turn out to be diagonal in the set of "pointer states" [1] which are selected by the crucial help of the interaction Hamiltonian $\left(H_{\text {int }}\right)$. They are stable subjected to the action of $H_{\text {int }}$, i.e., the interaction between the system and the environment will leave them unperturbed. That's exactly what makes them a "preferred" basis. No matter how weak the coupling that prevents the system from being isolated, the evolution of an open quantum system is eventually plagued by nonunitary features like decoherence and dissipation. Decoherence, in particular, is a quantum effect whereby the system loses its ability to exhibit coherent behaviour. Nowadays, decoherence stands as a serious obstacle in quantum information processing.

Since the work of Berry [2], the notion of geometric phases (GPs) was shown to have important consequences for quantum systems. Berry demonstrated that quantum systems could acquire phases that are geometric in nature. He showed that, besides the usual dynamical phase, an additional phase that was related to the geometry of the space state was generated during an adiabatic evolution. The original idea, framed within the context of adiabatic and cyclic evolutions of isolated systems, has been generalized in various aspects. While many of this propo-

\footnotetext{
*lombardo@df.uba.ar

†paula@df.uba.ar
}

sitions have been centered around pure states, the need to address the issue of GPs for mixed states rapidly gained prominence fuelled by the promise of realizing quantum logic gates under realistic physical conditions. In that direction, in [3] , it was introduced an alternative definition of GPs for nondegenerate density operators based upon quantum interferometry. In [4], a kinematic description of the mixed state GP was given and its definition extended to degenerate density operators. Recently, in [5], GPs for an open quantum system were studied and, finally, it was shown that the above apparently different approaches were related in a unifying framework. The effect on GPs of differents types of decoherence sources, such as dephasing and spontaneous decay, has been analyzed [6]. Likewise, it has been shown how to generate a geometric phase through modifications solely on the reservoir that interacts with a small subsystem [7].

GPs are useful in the context of quantum computation as a tool to achieve fault tolerance. However, practical implementations of quantum computing are always done in the presence of decoherence. Thus, a proper generalization of the geometric phase for unitary evolution to that for nonunitary evolution is central in the evaluation of the robustness of geometric quantum computation. This generalization to nonunitary evolution has been done in [8], where a functional representation of GP was proposed, after removing the dynamical phase from the total phase acquired by the system under a gauge transformation.

The GP for a mixed state under nonunitary evolution is then defined as

$$
\begin{aligned}
\Phi & =\arg \left\{\sum_{k} \sqrt{\varepsilon_{k}(0) \varepsilon_{k}(\tau)}\left\langle\Psi_{k}(0) \mid \Psi_{k}(\tau)\right\rangle\right. \\
& \left.\times e^{-\int_{0}^{\tau} d t\left\langle\Psi_{k}\left|\frac{\partial}{\partial t}\right| \Psi_{k}\right\rangle}\right\},
\end{aligned}
$$

where $\varepsilon_{k}(t)$ are the eigenvalues and $\left|\Psi_{k}\right\rangle$ the eigenstates of the reduced density matrix $\rho_{\mathrm{r}}$ (obtained after tracing over the reservoir degrees of freedom). In the last definition, $\tau$ denotes a time after the total system completes 
a cyclic evolution when it is isolated from the environment. Taking the effect of the environment into account, the system no longer undergoes a cyclic evolution. However, we will consider a quasicyclic path $\mathcal{P}: t \epsilon[0, \tau]$ with $\tau=2 \pi / \Omega$ ( $\Omega$ the system's frequency) [8]. It is worth noting that the phase in Eq.(1) is manifestly gauge invariant, since it only depends on the path in the state space, and that this expression, even though is defined for non degenerate mixed states, corresponds to the unitary geometric phase in the case that the state is pure (closed system) 3, 4].

It is expected that GPs can be only observed in experiments carried out in a time scale slow enough to ignore nonadiabatic corrections, but rapid enough to avoid the destruction of the interference pattern by decoherence [9]. So far, there has been no experimental observation of GPs for mixed states under nonunitary evolutions. The purpose of this letter is to study how GPs are affected by decoherence. Not only shall we analyze the effect of the environment on the GPs and their robustness against decoherence but also, under which conditions GPs can be measured. With these motivations, we shall introduce a "simplified" spin boson model and calculate the corrections to the unitary geometric phase for different physical environments. In the end, we shall estimate the decoherence times after which GPs can be no longer measured because they, literally, disappear.

The paper is organized as follow. In Section II we introduce the spin-boson model and we show the exact master equation for the reduced density matrix. We explicetly evaluate, in Section III, the corrections to the GPs induced by different types of environment and Section IV contains the calculation of the decoherence times. Finally, in Section V, we make our final remarks and comments.

\section{THE SPIN-BOSON MODEL}

Hereafter, we shall study an exact model for a twostate quantum system [or quantum bit (qubit)] coupled to a thermal bath of harmonic oscillators, where decoherence is the only effect on the system-particle. This is a particular case of the spin-boson model of Ref. 10] (where the tunnelling bare matrix element is $\Delta=0$ ) and has been used by many authors to model decoherence in quantum computers 11] and, in particular, it is extremely relevant to the proposal for observing GPs in a superconducting nanocircuit [12. We will concentrate on ohmic and supraohmic environments (at high, low, and zero temperature) coupled to the system spin. The Hamiltonian that describes the complete evolution of the two-state system interacting with the external environment is:

$$
H_{\mathrm{SB}}=\frac{1}{2} \hbar \Omega \sigma_{z}+\frac{1}{2} \sigma_{z} \sum_{n} \lambda_{n} q_{n}+\sum_{n} \hbar \omega_{n} a_{n}^{\dagger} a_{n},
$$

where the environment is described as a set of harmonic oscillators with a linear coupling in the oscillator coordinate. The interaction between the two-state system and the environment is entirely represented by a Hamiltonian in which the coupling is only through $\sigma_{z}$. A coupling of this form indicates that the state of the environment will be sensitive to the values of $\sigma_{z}$, which means that the environment can "observe" the value of $\sigma_{z}$ (i.e, where the system is $\mid \uparrow>$ or $\mid \downarrow>)$. The reason for this type of coupling is that the effects of a coupling proportional to $\sigma_{x}$ and/or $\sigma_{y}$ can be completely taken into account by the renormalization of the natural frequency of the system. However, in this particular case, $\left[\sigma_{z}, H_{\text {int }}\right]=0$ and the corresponding master equation is much simplified, with no frequency renormalization and dissipation effects. If we assume that (i) the system and the environment are initially uncorrelated and (ii) the environment is initially in thermal equilibrium at temperature $T$ (or the vacuum for zero temperature), the master equation for the reduced density matrix is

$$
\dot{\rho}_{\mathrm{r}}=-i \Omega\left[\sigma_{z}, \rho_{\mathrm{r}}\right]-\mathcal{D}(t)\left[\sigma_{z},\left[\sigma_{z}, \rho_{\mathrm{r}}\right]\right]
$$

In other words, the model describes a purely decohering mechanism, solely containing the diffusion term $\mathcal{D}(t)$, where no energy exchange between system and bath is present. The diffusion coefficient is given by

$$
\mathcal{D}(t)=\int_{0}^{t} d s \int_{0}^{\infty} d \omega J(\omega) \cos (\omega s) \operatorname{coth}\left(\frac{\beta \hbar \omega}{2}\right),
$$

where $\beta=1 / k_{B} T\left(k_{B}\right.$ is Boltzman constant) and $J(\omega)$ is the spectral density of the environment defined by the expression $J(\omega)=\sum_{n} \lambda_{n}^{2} \delta\left(\omega-\omega_{n}\right) / 2 m_{n} \omega_{n}$. One assumption we shall make throughout this paper is that $J(\omega)$ is a reasonably smooth function of $\omega$, and that is of the form $\omega^{n}$ up to some frequency $\Lambda$ that may be large compared to $\Omega$. In particular, the case with $n=1$ is the "ohmic" case and the one with $n>1$ is the "supraohmic" case. It is easy to see in Eq.(4) that the system's dynamics depends crucially both on the external temperature and on the type of environment.

\section{GEOMETRIC PHASE FOR THE COMPOSITE SYSTEM}

We shall compute the geometric phase for the spinboson model. It is easy to check that $\rho_{\mathrm{r}_{01}}(t)=$ $e^{-i \Omega t-\Gamma(t)} \rho_{\mathrm{r}_{01}}(0)$ is solution of the master equation (Eq.(3)) with $\Gamma(t)=\int_{0}^{t} d t^{\prime} \mathcal{D}\left(t^{\prime}\right)$ and $\rho_{\mathrm{r}_{01}}(0)$ a constant determined by initial conditions. Here we are implying that the diagonal terms do not evolve in time, i.e. $\rho_{\mathrm{r}_{\mathrm{ii}}}(t)=\rho_{\mathrm{r}_{\mathrm{ii}}}(0)$, for $i=0,1$ (the spin state basis), assuming the population dynamics of the system is essentially frozen on the time scales of interest [11]. The decoherence factor is then given by

$$
\Gamma(t)=4 \int_{0}^{\infty} d \omega J(\omega) \operatorname{coth}\left(\frac{\beta \hbar \omega}{2}\right) \frac{(1-\cos (\omega t))}{\omega^{2}} .
$$


When a specific choice turns out to be useful, we shall assume the following functional form for the spectral density $J(\omega)=\gamma_{0} / 4 \omega^{n} \Lambda^{n-1} e^{-\omega / \Lambda}\left[10\right.$, where $\gamma_{0}$ is the dissipative constant (in suitable units) and $\Lambda$ is the cutoff frequency.

As the factor $\Gamma$ is real, unitarity requires $\rho_{\mathrm{r}_{01}}(0)=$ $\rho_{\mathrm{r}_{10}}(0)$ since $\rho_{\mathrm{r}_{10}}=\rho_{\mathrm{r}_{01}}{ }^{*}$. For a spin $1 / 2$ system, the state space consists of all points on and inside the Bloch sphere. Then, we assume that the system is initially in the Bloch state

$$
|\Psi(0)\rangle=\cos \left(\theta_{0} / 2\right)|e\rangle+\sin \left(\theta_{0} / 2\right)|g\rangle .
$$

The constants are determined so that $\rho_{\mathrm{r}}(0)=$ $|\Psi(0)\rangle\langle\Psi(0)|$. Then, for times $t>0$, the reduced density matrix is

$$
\rho_{\mathrm{r}}(t)=\left(\begin{array}{cr}
\cos \left(\theta_{0} / 2\right)^{2} & 1 / 2 \sin \left(\theta_{0}\right) e^{i \Omega t-\Gamma(t)} \\
1 / 2 \sin \left(\theta_{0}\right) e^{-i \Omega t-\Gamma(t)} & \sin \left(\theta_{0} / 2\right)^{2}
\end{array}\right) .
$$

The eigenvalues of the above reduced density matrix are easily calculated:

$$
\varepsilon_{ \pm}(t)=\frac{1}{2} \pm \frac{1}{2} \sqrt{\cos \left(\theta_{0}\right)^{2}+\exp (-2 \Gamma(t)) \sin \left(\theta_{0}\right)^{2}} .
$$

In order to estimate the geometric phase, we only need the eigenvector $\left|\Psi_{+}(t)\right\rangle$ since $\varepsilon_{-}(0)=0$, and, hence, the only contribution to the phase comes from that eigenvector and its corresponding eigenvalue (see Eq.(10). Then, we write it here as:

$$
\left|\Psi_{+}(t)\right\rangle=e^{-i \Omega t} \sin \left(\theta_{t} / 2\right)|e\rangle+\cos \left(\theta_{t} / 2\right)|g\rangle,
$$

with $\tan \left(\theta_{t} / 2\right)=\exp (-\Gamma(t)) \cot \left(\theta_{0} / 2\right)$. It is easy to check that, when $\Gamma=0$, we reobtain the results for the unitary case. Hence, once we have the eigenvalues and eigenvectors, we can calculate the factor $\left\langle\Psi_{k} \mid \dot{\Psi}_{k}\right\rangle$ of Eq. (11). Performing the time derivatives, we get $\left\langle\Psi_{k} \mid \dot{\Psi}_{k}\right\rangle=$ $-i \Omega \sin \left(\theta_{t} / 2\right)^{2}$, therefore, the geometric phase is

$$
\Phi=\arg \left\{\sqrt{\varepsilon_{+}(\tau) \varepsilon_{+}(0)}\left\langle\Psi_{+}(\tau) \mid \Psi_{+}(0)\right\rangle e^{i \Omega \int_{0}^{\tau} d t \sin \left(\frac{\theta_{t}}{2}\right)^{2}}\right\} .
$$

This result holds for any density matrix has these eigenvalues and eigenvectors, independently of the exact expression of $\Gamma$, as long as $\varepsilon_{-}(0)=0$. By inserting Eqs. (6) and (7) into the above expression, the geometric phase related to a quasicyclic path $\mathcal{P}: t \epsilon[0, \tau]$ (with $\tau=2 \pi / \Omega$ [8]) is (assuming $\cos \left(\theta_{0} / 2\right) \geq 0$ ):

$$
\Phi=\Omega \int_{0}^{\tau} d t \sin \left(\frac{\theta_{t}}{2}\right)^{2} .
$$

In order to evaluate this integral, we will perform a serial expansion in terms of powers of the dissipative constant $\gamma_{0}$, and, consequently, the unitary phase $\Phi^{U}$ is corrected by the presence of the environment as:

$$
\begin{aligned}
\Phi & =\Phi^{U}+\delta \Phi \approx \pi\left(1-\cos \left(\theta_{0}\right)\right) \\
& +\left.\gamma_{0} \frac{\Omega}{2} \sin \left(\theta_{0}\right)^{2} \cos \left(\theta_{0}\right) \int_{0}^{\tau} d t\left[\frac{\partial \Gamma(t)}{\partial \gamma_{0}}\right]\right|_{\gamma_{0}=0}
\end{aligned}
$$

where we can see that the first term of the expansion is the solution we would have obtained if the evolution would have been unitary, i.e $\Phi^{U}=\pi\left(1-\cos \left(\theta_{0}\right)\right)$. If, for example, we assume an ohmic $(n=1)$ environment at high temperature, then the spectral density takes the particular form $J(\omega)=\gamma_{0} / 4 \omega e^{-\omega / \Lambda}$. In that case, $\hbar \omega<<2 k_{B} T$, we can approximate $\operatorname{coth}(\beta \hbar \omega / 2)$ in Eq. (4) by $2 k_{B} T /(\hbar \omega)$ and the decoherence coefficient $\Gamma$ becomes $\Gamma=\left(\gamma_{0} \pi k_{B} T\right) t / \hbar$. Then, the geometric phase is corrected by:

$$
\delta \Phi=\pi^{2} \gamma_{0}\left(\frac{\pi k_{B} T}{\hbar \Omega}\right) \sin \left(\theta_{0}\right)^{2} \cos \left(\theta_{0}\right) .
$$

This correction to the unitary phase is proportional to $\gamma_{0} k_{B} T / \hbar \Omega$. Then, it is bigger for hotter environments and can not be neglected. It is worth mentioning that, formally, our solution is similar to the one proposed by authors in [13] where they made no a priori assumption about the dynamics of the system. On the contrary, our solution is obtained from scratch and can be applied to different environments at any temperature. What's more, this correction is more realistic since they assumed a very much simplified environment.

If we were to assume the same ohmic environment but at zero temperature, then the phase would be corrected in a significantly different way, as one would expect since there is one time scale lost. In that case, the factor $\operatorname{coth}(\beta \hbar \omega / 2)$, in the definition of $\Gamma(t),($ Eq. (4id) can be approximated by 1 and the correction obtained is:

$$
\delta \Phi=\pi \gamma_{0}\left(-1+\log \left(\frac{2 \pi \Lambda}{\Omega}\right)\right) \sin \left(\theta_{0}\right)^{2} \cos \left(\theta_{0}\right) .
$$

It is worthy noting that this correction comes from the zero point fluctuations of the environment.

Beyond the commonly assumed ohmic spectrum for the bath, generic nonohmic environments can be studied with this model. Owing to the ultrashort time bath correlations, nontrivial short-time system dynamics enters. What's most appealing about this case is the fact that the electromagnetic field can be modeled by a supraohmic environment which results very useful for quantum optics and trying to measure the geometric phases. In the following, we shall evaluate the correction of the geometric phases in the presence of this kind of environments.

For high temperature, we can still make the same approximation than in the ohmic case, and then, the estimation of the $\Gamma$ factor is straightforward. Using the spectral density with $n=3$, the correction to the unitary phase is:

$$
\delta \Phi=\pi \gamma_{0}\left(\frac{2 k_{B} T}{\hbar \Lambda}\right) \sin \left(\theta_{0}\right)^{2} \cos \left(\theta_{0}\right) .
$$

We can also estimate the correction to the unitary phase in the presence of an supraohmic environment but at zero temperature as:

$$
\delta \Phi=\pi \gamma_{0} \sin \left(\theta_{0}\right)^{2} \cos \left(\theta_{0}\right) .
$$


As $\Lambda$ is the maximum available frequency in the environment, a valid assumption is that $\Omega \lesssim \Lambda$. In that case, we can see that the parenthesis in Eq.(10) is of order one and, then, both corrections at zero temperature (Eq. (10) and Eq.(12)), for the ohmic and nonohmic case, are similar. The same occurs for high temperate in Eq. (9) and Eq.(11). These results are remarkably interesting and enhance the robustness of the model (and of the geometric phase).

\section{DECOHERENCE TIMES FOR THE COMPOSITE SYSTEM}

In this section we shall estimate the decoherence time, i.e. the time-scale when the nondiagonal terms of the reduced density matrix are suppressed. Therefore, it is a very important time scale to take into account when planning an experiment to measure the geometric phases.

Let's take for example an interference experiment. The experiment starts by the preparation of two wave packets in a coherent superposition, assuming each of the particles follows a well defined classical path $\left(C_{1}\right.$ and $C_{2}$, respectively), as $\Psi(t=0)=\left(\varphi_{1}(x)+\varphi_{2}(x)\right) \otimes \chi_{0}(y)$, where $\chi_{0}(\vec{y})$ represents the initial quantum state of the environment (whose set of coordinates is denoted by $\vec{y}$ ). Due to the interaction between the system and the environment, the total wave function at a later time $t$ is $\Psi(t)=\varphi_{1}(\vec{x}, t) \otimes \chi_{1}(\vec{y}, t)+\varphi_{2}(\vec{x}, t) \otimes \chi_{2}(\vec{y}, t)$. It is easy to note that the states $\varphi_{1}$ and $\varphi_{2}$ became entangled with two different states of the environment. Therefore, the density matrix of the system at time $t$ (for example when interference pattern is examined) is,

$$
\begin{aligned}
\rho_{\mathrm{r}}\left(x, x^{\prime}\right) & =\varphi_{1}^{*}(x) \varphi_{1}\left(x^{\prime}\right)+\varphi_{2}^{*}(x) \varphi_{2}\left(x^{\prime}\right) \\
& +\left(\varphi_{1}^{*}(x) \varphi_{2}\left(x^{\prime}\right)+\varphi_{2}^{*}(x) \varphi_{1}\left(x^{\prime}\right)\right) \mathcal{F}(t) .
\end{aligned}
$$

The last two terms in the above expression represent the quantum interferences. The overlap factor $\mathcal{F}(t)$ 14 encodes the information about the statistical nature of noise since it is obtained by tracing over the degrees of freedom of the bath. Hence, it produces a decaying term that tends to eliminate the interference pattern. Therefore, noise makes $\mathcal{F}(t)$ less than one, and the goal is to quantify how it slightly destroys the particle interference pattern. In the case of the exact system we are studying here, $\mathcal{F}(t)=e^{-\Gamma(t)}$. When $\Gamma$ is big, the off-diagonal terms (coherences) elements will vanish in a short time scale, and hence, the open system will not acquire GP. Then, in order to know the time-scale at which the quantum interferences are suppressed, we have to estimate the "decoherence" time $t_{D}$ for each of the four cases studied above, by setting $\Gamma\left(t_{D}\right) \approx 1$.

For the ohmic environment, the decoherence factor is $\Gamma^{1 T}=\left(k_{B} T \pi \gamma_{0} / \hbar\right) t$ in the limit of high temperature. Then, the decoherence time scale for this case is, in principle, extremely short, estimated as $t_{D}^{1 T}=\hbar /\left(k_{B} T \pi \gamma_{0}\right)$
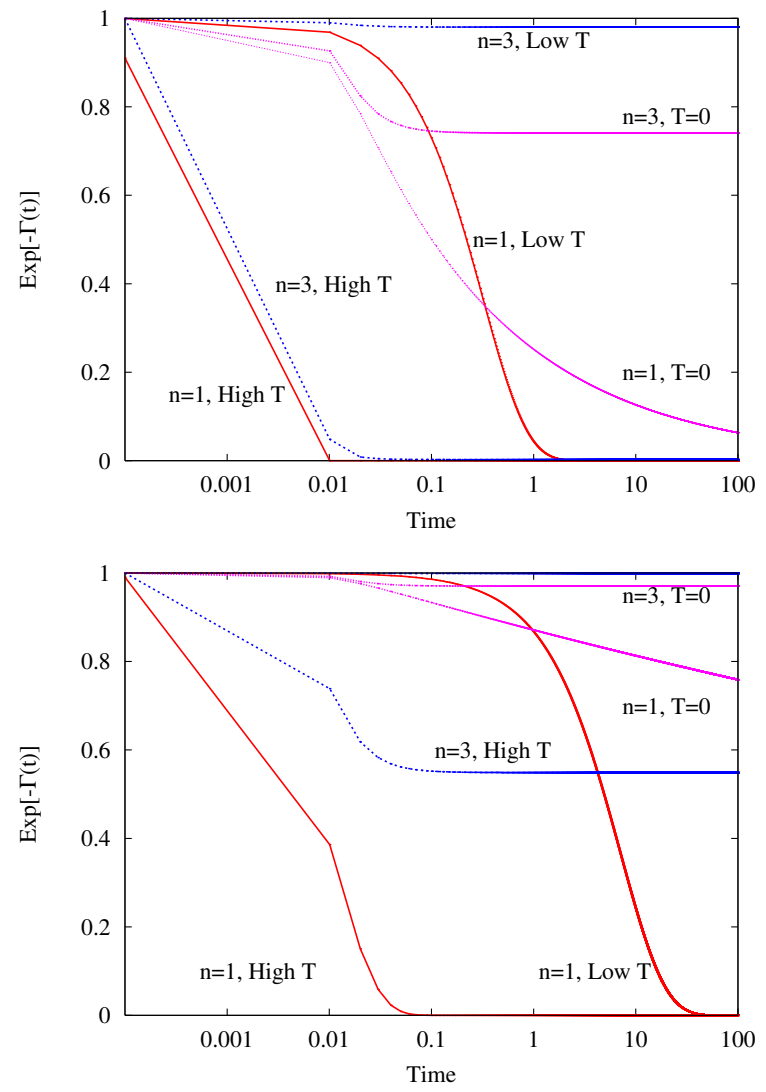

FIG. 1: Comparison between the $\Gamma(t)$ coefficients for the ohmic case $(n=1)$ and the supraohmic case $(n=3)$ for different environments for high $(T=1000)$, low $(T=1.55)$ and zero temperature $\left(\hbar=1=k_{B}\right) . \Lambda=100 \Omega$ and $\gamma_{0}=0.3$ (top) and $\gamma_{0}=0.03$ (bottom). Time is measured in units of $\Omega$. It is easy to note that the low temperature limit is not zero temperature.

(see Fig 1). All other cases depend explicitly upon the cutoff. For the ohmic environment at zero temperature, the decoherence factor is $\Gamma^{10}=\gamma_{0} / 2 \log \left(1+\Lambda^{2} t^{2}\right)$. For times $\Lambda t \geq 1$, the decoherence time scales as $t_{D}^{10}=$ $e^{1 / \gamma_{0}} / \Lambda$. Sooner or later, decoherence is always present in this environment as well. As it is clearly shown in Fig 1 decoherence is delayed as $\gamma_{0}$ decreases (bottom). Things are slightly different for the supraohmic environment. In the high temperature limit, the decoherence factor is $\Gamma^{3 T}=\left(2 k_{B} T \gamma_{0}\right) \Lambda t^{2} / \hbar$ for $\Lambda t \ll 1$. Then, the estimation of the decoherence time is straightforward $t_{D}^{3 T}=1 / \Lambda \sqrt{\hbar \Lambda /\left(2 k_{B} T \gamma_{0}\right)}$. However, if $\Lambda t \geq 1$, $\Gamma^{3 T}=2 k_{B} T \gamma_{0} /(\hbar \Lambda)$, constant in time. Then, decoherence shall occur if and only if $2 k_{B} T \gamma_{0} \gg \hbar \Lambda$, and that shall happen in a time $t<1 / \Lambda$ as it is shown on top of Fig 1 At the bottom of Fig $1 \gamma_{0}$ is smaller and then, $k_{B} T \gamma_{0}<\hbar \Lambda$, which means that $\Gamma^{3 T}$ will never be of order one and, therefore, decoherence shall not be effective. 
Finally, in the supraohmic case at zero temperature, the decoherence factor is $\Gamma^{30}=\gamma_{0} \Lambda^{4} t^{4} /\left(1+\Lambda^{2} t^{2}\right)^{2}$. We can see in Fig प that decoherence never occurs for this case. This is so because $\Gamma^{30}=\gamma_{0}$ for $\Lambda t \geq 1$. The decoherence factor will be a constant value of $e^{-\gamma_{0}}$ for all times. Since $\gamma_{0}<1$, this factor will never be of order one. In conclusion, as high-T environments are so efficient producing decoherence, there is a strong condition over the dissipative constant $\gamma_{0}$ in order to get $t_{D}>\tau$ 15, i.e. $\gamma_{0}<\hbar \Lambda / k_{B} T$, which actually is unfeasible. Bigger $\gamma_{0}$ implies shorter decoherence times. However, for zero$\mathrm{T}$ baths, the condition on $\gamma_{0}$ relaxes to $\gamma_{0}<1$ making $t_{D}>\tau$ and allowing experimental observation.

\section{FINAL REMARKS}

When measuring a GP, the dynamical phase has to be eliminated. It can be either canceled, for example, using spin-echo technique for spins in magnetic fields [16], or one can parallel transport the state vector in order to ensure that the dynamical phase is zero at all times. Even though the state does not acquire a phase locally, it can acquire a phase globally after completing a cyclic evolution. This global phase is equal to the geometric phase. GPs are useful in the context of quantum computation as a tool to achieve fault tolerance. However, practical implementations of quantum computing are always done in the presence of decoherence, a nonunitary effect of open systems. However, there have been many assumptions about the time scale at which the geometric phase can be measured, there has been no reported observation yet for mixed states under nonunitary evolutions.

It has been argued that the observation of GPs should be done in times long enough to obey the adiabatic approximation but short enough to prevent decoherence from deleting all phase information. In this article, not only have we shown an exact model where the correction to the GPs can be evaluated for different types of environment at any temperature but also, estimations of the corresponding times at which the interference pattern is mostly reduced and decoherence becomes effective. This model allows us to evaluate an exact master equation for the reduced density matrix and, as a consequence, to elaborate a full analysis on the effect of decoherence on the geometric phase of the composite system. The goal is the analysis of the effect produced by decoherence, which should be essential to design experimental setups in order to observe geometric phases using, for example, interferometry. We stressed that the convenient environment to observe GPs is at zero temperature in the underdamped limit and that these decoherence times should be taken into account when extending the study of GPs to the nonunitary regime, especially pertinent to the application to fault-tolerant quantum computation [17].

\section{ACKNOWLEDGMENTS}

We thanks F. Mazzitelli for useful comments. This work was supported by UBA, CONICET, and ANPCyT, Argentina.
[1] W. Zurek, Rev. Mod. Phys.75, 715 (2001).

[2] M.V. Berry, Proc. R. Soc. Lond. A 392, 45 (1984).

[3] E. Söqvist, A.K. Pati, A. Ekert, J.S. Anandan, M. Ericsson, D.K.L. Oi, and V. Vedral, Phys. Rev. Lett. 85, 2845 (2000).

[4] K. Singh, D.M. Dong, K. Basu, J.L. Chen, and J.F. Du, Phys. Rev. A, 67, 032106 (2003).

[5] A.T. Rezakhani and P. Zanardi, Phys. Rev. A 73, 012107 (2006).

[6] A. Carollo, I. Fuentes-Guridi, M. Franca Santos, and V. Vedral, Phys. Rev. Lett. 90, 160402 (2003); Phys. Rev. Lett. 92, 020402 (2004).

[7] A. Carollo, M. Franca Santos, and V. Vedral, Phys. Rev. Lett. 96, 020403 (2006); A. Carollo, G. M. Palma, A. Lozinski, M. Frana Santos, and V. Vedral, Phys. Rev. Lett. 96, 150403 (2006).

[8] D. M. Tong, E. Sjqvist, L. C. Kwek, and C. H. Oh, Phys. Rev. Lett. 93, 080405 (2004).

[9] R.S. Whitney, Y. Makhlin, A. Shnirman, and Y. Gefen, Phys. Rev. Lett. 94, 070407 (2005).

[10] A.J. Leggett, S. Chakravarty, A.T. Dorsey, M.P.A.
Fisher, A. Garg, and W. Zwerger, Rev. Mod. Phys. 59, 1 (1987).

[11] G.M. Palma, K. Suominen, and A. Ekert, Proc. R. Soc. London, Ser. A 452, 567 (1996); L. Viola and S. Lloyd, Phys. Rev. A 58, 2733 (1998).

[12] G. Falci, R. Fazio, G.M. Palma, J. Siewert, and V. Vedral, Nature (London) 407, 355 (2000).

[13] X.X. Yi, D. M. Tong, L. C. Wang, L. C. Kwek, and C. H. Oh, Phys. Rev. A 73, 052103 (2006).

[14] F.C. Lombardo, F.D. Mazzitelli, and P.I. Villar, Phys. Rev. A 72, 042111 (2005); F.C. Lombardo and P.I. Villar, J.Phys. A 39, 6509 (2006).

[15] A.T. Rezakhami and P. Zanardi, Phys. Rev. A 73, 052117 (2006).

[16] A. Ekert et al., J. Mod. Opt. 47, 2501 (2000).

[17] J. Du, P. Zou,M. Shi, L.C. Kwek,J. Pan, C.H. Oh, A. Ekert, D. K.L. Oi, and M. Ericsson, Phys. Rev. Lett. 91, 100403 (2003); I. Fuentes-Guridi, E.R. Livine, Phys. Rev. Lett. 94, 020503 (2005). 